Annuaire suisse de politique de développement

22-2 | 2003

Société de l'information et coopération internationale

\title{
Helvetas : vers des technologies participatives
}

\section{Marc Steinlin}

\section{(2) OpenEdition}

\section{Journals}

Édition électronique

URL : http://journals.openedition.org/aspd/569

ISSN : 1663-9669

Éditeur

Institut de hautes études internationales et du développement

Édition imprimée

Date de publication : 1 novembre 2003

Pagination : 141-143

ISSN : 1660-5934

Référence électronique

Marc Steinlin, «Helvetas : vers des technologies participatives », Annuaire suisse de politique de développement [En ligne], 22-2 | 2003, mis en ligne le 22 mars 2010, consulté le 07 septembre 2020. URL : http://journals.openedition.org/aspd/569 


\section{Helvetas: vers des technologies participatives}

\section{Marc Steinlin*}

\section{Les TIC, nouvelles perspectives pour le développement}

Au départ, les technologies de l'information et de la communication (TIC) ont servi de terrain d'expérimentation à des pionniers pleins d'imagination, qui allaient les introduire ainsi que leurs applications dans le champ de la coopération au développement. Dans les années 1990, les grands acteurs (organisations internationales de l'ONU, agences bilatérales comme l'USAID) ont les premiers abordé la question des TIC dans les débats sur le développement.

En 1999, Helvetas a dû se positionner sur cette question dans le cadre d'un mandat de la Banque mondiale. La place réservée aux TIC ressortait clairement de son programme sectoriel World Link for Development. On a aussi très vite compris qu'il ne s'agissait pas ici que de l'ouverture d'un champ d'activités prometteur, mais de défis et de questions qu'Helvetas devait s'atteler à relever activement.

L'organisation allait néanmoins se retrouver partagée. D'un côté, elle reconnaissait l'importance de ces nouvelles technologies: immense potentiel de développement, solutions nouvelles à de vieux problèmes, possibilités de croissance pour les pays du Sud comme pour l'organisation Helvetas elle-même. De l'autre, une certaine incertitude prévalait, suscitée par la nouveauté de la question, le manque d'information et d'expérience, la notion émergente de «fracture numérique», la crainte aussi de répéter dans de nouveaux domaines les mêmes anciennes erreurs en matière de développement - syndrome des «tracteurs rouillant sous le soleil», c'est-à-dire des projets présentant une composante matérielle importante mais sans processus de développement durable.

Mise au défi, Helvetas a réagi par une phase de réflexion, notamment en réalisant en 1999 une étude modèle sur les TIC au Mali. Elle s'est appuyée sur celle-ci pour définir ses premières orientations et lignes directrices; elle a cherché des niches pour les intégrer à son programme général. Telle est la genèse de la stratégie d'Helvetas en matière de TIC.

\section{Stratégie d'Helvetas: quelle place pour les TIC?}

Les TIC modifient la pratique de l'aide au développement à double titre: d'une part, ces technologies obligent la coopération au développement à répondre à de nouveaux défis. Des problèmes et des contextes différents requièrent un cadre d'activités différent. D'autre part, les TIC élargissent les horizons et offrent la possibilité d'appréhender autrement, par d'autres voies, d'anciens problèmes, souvent restés sans réponses. 
Dans le cadre d'un processus d'empowerment, la prise de conscience de la révolution globale de l'information et de la communication est fondamentale. Pour pouvoir organiser librement, sur un plan local, son existence, il importe qu'un individu ou une communauté dispose de connaissances, d'une information et d'une capacité d'évaluer ces processus de changement au niveau global.

Dans tous les cas, l'accès aux moyens d'information reste essentiel. En tant que «technologies participatives», les TIC peuvent notamment contribuer d'une manière substantielle à intégrer certains groupes marginalisés, par exemple dans le cadre de conseils aux paysans. De même, dans un processus de démocratisation et de décentralisation, elles pourront à l'avenir s'avérer très utiles pour promouvoir la conscientisation des populations et le dialogue. Enfin, elles ouvrent des possibilités intéressantes pour collecter et diffuser les savoirs locaux, mais aussi pour les mettre en interaction avec les savoirs modernes et globaux, ce qui exigera une redéfinition du knowledge management. Il faudra dès lors considérer les TIC comme des éléments vectoriels dans tous les projets où elles peuvent apporter une plus-value.

Helvetas a toutefois aussi fait des TIC un sous-secteur de ses activités. Elle entend leur accorder une attention particulière dans le domaine de la formation. Dans cette perspective, Helvetas a estimé qu'il fallait ajouter le travail sur ordinateur au nombre des compétences culturelles de base, avec la lecture, l'écriture et le calcul. Les TIC font donc ainsi partie intégrante de l'éducation formelle. Celleci peut être considérée sous quatre angles particuliers: la computer lite- racy (surtout les connaissances conceptuelles), la pédagogie, l'enseignement et, enfin, le développement de programmes didactiques. Devant l'ampleur de la tâche qui doit amener les pays du Sud à se familiariser avec les TIC, Helvetas souligne également l'importance de l'éducation informelle; elle s'appuie ici sur le moteur intrinsèque du développement, c'està-dire essentiellement sur le plaisir de la découverte et l'envie d'apprendre d'individus capables d'innovation. Helvetas a développé ainsi l'idée des «infotopes» - sur le modèle des biotopes. L'infotope est donc un lieu d'échange de connaissances et d'informations, pour une confrontation concrète avec les TIC. Les ordinateurs et Internet, champ d'expérimentation, doivent être rendus aussi librement accessibles que possible au groupe cible. En outre, il s'agira aussi d'évaluer et de développer de nouvelles méthodes didactiques et de connexion.

D’une manière générale, la stratégie TIC d'Helvetas doit s'insérer dans les stratégies et principes plus larges existants. S'appuyant sur des lacunes et des erreurs constatées chez d'autres acteurs, Helvetas a créé le concept des quatre wares, qui soustendra ses activités: à côté du hardware et du software, elle a défini les notions de frameware et de brainware. C'est sur ces deux dernières qu'elle a concentré ses réflexions. Le frameware concerne les aspects systémiques des TIC: conditions-cadres légales et sociales, aspects organisationnels et institutionnels (organisational development), accès aux TIC (y compris au sens social), problèmes d'entretien, gestion des savoirs, etc. Le brainware concerne les ressources humaines, les aspects de la forma- 
tion, les questions pédagogiques. Pour durer, les projets devront trouver un équilibre entre les quatre domaines.

\section{L'intérêt concret des TIC aux yeux d'Helvetas}

Trois exemples illustrent le travail d'Helvetas dans ce domaine. Dans le cadre d'une coopération déjà ancienne avec les séminaires d'enseignants au Bhoutan, Helvetas a intégré les TIC directement dans la formation. D'un côté, les futurs enseignants devront utiliser les TIC pour leur propre travail professionnel; de l'autre, ils devront être bientôt capables d'enseigner les TIC comme matière en soi. Cela suppose qu'un certain nombre de conditions prévalent: que les enseignants exploitent les infrastructures techniques de manière durable, qu'ils se soient approprié les connaissances pédagogiques et les concepts nécessaires; enfin, que l'accès libre des étudiante-s aux ordinateurs soit garanti et réglementé.

Au Paraguay, les TIC deviendront le principal instrument de la vulgarisation agricole et paysanne. Les conseillers seront munis d'ordinateurs portables et mis en réseau. Dans le cadre du développement du knowledge management, ils s'entraideront directement sur le terrain via Internet. Par la suite, les paysans devraient être également reliés entre eux pour des échanges d'informations au niveau local.

Dans le cadre de la décentralisation de l'administration publique au Mali, il est prévu de mettre en réseau via Internet des services régionaux de conseil pour les administrations communales. Celles-ci pourront alors échanger des connaissances, des informations et d'autres ressources (par exemple des documents administratifs); enfin, le réseau permettra, à plus long terme, l'introduction d'éléments de e-governance. Un développement particulièrement intéressant lorsque la communication et l'interaction par les voies traditionnelles sont quasiment impossibles.

Enfin, il ne faut pas oublier l'influence déterminante des TIC sur la communication au sein même de l'organisation Helvetas. L'installation d'un réseau interne Intranet a permis le rapprochement de ses propres experts et rendu plus disponibles et facilement utilisables leurs compétences disséminées dans le monde. 